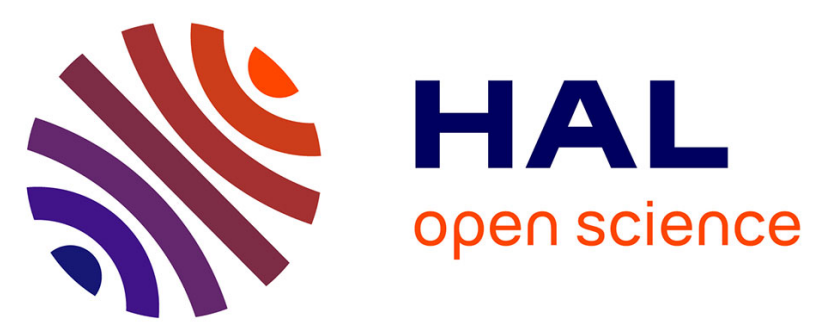

\title{
Science with GYES: a multifibre high-resolution spectrograph for the prime focus of the Canada-France-Hawaii Telescope
}

P. Bonifacio, F. Arenou, C. Babusiaux, C. Balkowski, O. Bienaymé, D. Briot, E. Caffau, R. Carlberg, B. Famaey, P. François, et al.

\section{To cite this version:}

P. Bonifacio, F. Arenou, C. Babusiaux, C. Balkowski, O. Bienaymé, et al.. Science with GYES: a multifibre high-resolution spectrograph for the prime focus of the Canada-France-Hawaii Telescope. SPIE Astronomical Telescopes + Instrumentation, Jun 2010, San Diego, United States. 77350E (9p.), $10.1117 / 12.857081$. hal-00527598

\section{HAL Id: hal-00527598 \\ https://hal.science/hal-00527598}

Submitted on 22 Jul 2021

HAL is a multi-disciplinary open access archive for the deposit and dissemination of scientific research documents, whether they are published or not. The documents may come from teaching and research institutions in France or abroad, or from public or private research centers.
L'archive ouverte pluridisciplinaire HAL, est destinée au dépôt et à la diffusion de documents scientifiques de niveau recherche, publiés ou non, émanant des établissements d'enseignement et de recherche français ou étrangers, des laboratoires publics ou privés. 


\title{
Science with GYES: A multi-fibre high resolution spectrograph for the prime focus of the Canada-France-Hawaii Telescope
}

\author{
Bonifacio P. ${ }^{a}$, Arenou F. ${ }^{a}$, Babusiaux C. ${ }^{a}$, Balkowski C. ${ }^{a}$, \\ Bienaymé O. ${ }^{b}$, Briot D. ${ }^{a}$, Caffau E. ${ }^{a}$, Carlberg R. ${ }^{c}$, Famaey B. ${ }^{b}$, \\ François P. ${ }^{a}$, Frémat Y. ${ }^{d}$, Gómez A. ${ }^{a}$, Haywood M. ${ }^{a}$, Hill V. ${ }^{e}$, Katz D. ${ }^{a}$. \\ Kudritzky R..$^{f}$, Lallement R..$^{g}$, de Laverny P. ${ }^{e}$, Lemasle B. ${ }^{h}$, Martayan C. ${ }^{i, a}$, \\ Monier R. ${ }^{k}$, Mourard D. ${ }^{k}$, Nardetto N. ${ }^{k}$, Recio Blanco A. ${ }^{e}$, Robichon N. ${ }^{a}$, \\ Robin A. C. ${ }^{j}$, Rodrigues M. ${ }^{a}$, Royer F. ${ }^{a}$, Soubiran C. ${ }^{l}$, Turon C. ${ }^{a}$, Venn K. ${ }^{m}$, \\ Viala Y. ${ }^{a}$

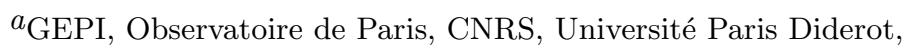 \\ Place Jules Janssen, 92190 Meudon, France; \\ ${ }^{b}$ Observatoire Astronomique de Strasbourg, \\ 11, rue de l'Université, F-67000 Strasbourg, France; \\ ${ }^{c}$ Department of Astronomy and Astrophysics, University of Toronto, \\ Toronto, Ontario M5S 3H4 Canada;

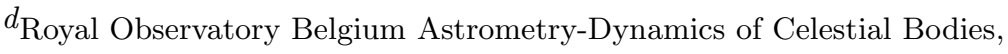 \\ Av Circulaire 3-Ringlaan 3, BE 1180 Brussels, Belgium; \\ ${ }^{e}$ Université de Nice Sophia Antipolis, CNRS, Observatoire de la Côte d'Azur, Laboratoire Cassiopée, \\ B.P. 4229, 06304 Nice Cedex 4, France;

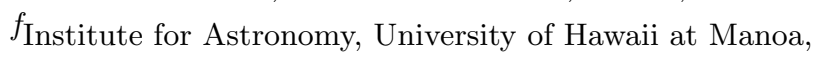 \\ 2680 Woodlawn Drive, Honolulu, Hawaii 96822, USA;

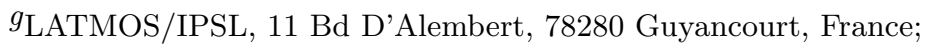 \\ $h_{\text {Kapteyn Astronomical Institute, }}$ \\ Landleven 12, 9747 AD Groningen, The Netherlands; \\ ${ }^{i}$ European Southern Observatory, \\ Alonso de Còrdova, 3107 Vitacura - Santiago, Chile; \\ $j_{\text {Observatoire de Besançon, }}$ \\ 41 bis, avenue de l'Observatoire, B.P. 1615, 25010 Besançon Cedex, France; \\ ${ }^{k}$ Université de Nice Sophia Antipolis, CNRS, Observatoire de la Côte d'Azur, Laboratoire Fizeau, \\ B.P. 4229, 06304 Nice Cedex 4, France; \\ lObservatoire de Bordeaux, \\ 2 Avenue de l'Observatoire, F-33270 Floirac, France; \\ $m_{\text {Department of Physics \& Astronomy The University of Victoria }}$ \\ Elliott Building, 3800 Finnerty Road Victoria, BC, V8P 5C2, Canada
}

September 19, 2010

Abstract
We present the scientific motivations for GYES: a high multiplex (of the order of several hundred), high 
resolution (about 20000), spectrograph to be placed at the prime focus of the CFHT. The main purpose of such an instrument is to conduct a spectroscopic survey complementary to the Gaia mission. The final Gaia catalogue (expected around 2020) will provide accurate distances, proper motions and spectrophotometry for all the stars down to a magnitude of 20. The spectroscopic instrument on board the Gaia satellite will provide intermediate resolution $(\mathrm{R}=11500)$ spectra for stars down to the $17^{\text {th }}$ magnitude. For the fainter stars there will be no radial velocity information. For all the stars the chemical information will be limited to a few species. A multifibre spectrograph at the prime focus of the CFHT will be able to provide the high resolution spectra for stars fainter than $13^{\text {th }}$ magnitude, needed to obtain both accurate radial velocities and detailed chemical abundances. The possible use of GYES will not be limited to Gaia complementary surveys and we here describe the potentialities of such an instrument. We describe here how the scientific drivers are translated into technical requirements.

Keywords: Instrumentation: spectrographs, Galaxy: abundances, Galaxy: structure, ISM: abundances, ISM: kinematics, Galaxies: abundances, Galaxies: evolution, Galaxies: kinematics

\section{INTRODUCTION}

The launch of the Gaia satellite, scheduled in 2012, will represent a major milestone in astronomical research of the third millennium. The final Gaia catalogue (expected around 2020) will provide accurate distances, proper motions and spectrophotometry for all the stars down to a magnitude of 20. Such information will totally revolutionise our knowledge of the Galaxy and its stellar populations. We will be able to dissect the different Galactic components and confront this detailed structure with models of galaxy formation and their cosmological context. The full three dimensional velocity vector of a large number of stars, and their detailed chemical composition will allow to address crucial questions like the amount and distribution of dark matter in the Galaxy and the formation and evolution of the different Galactic components. Radial velocities and chemical abundances can be derived from high resolution spectra. On board the Gaia satellite there will be a spectroscopic instrument, the RVS, which will provide intermediate resolution ( $\mathrm{R}=11500$, for stars brighter than about 14 and $R=6500$, for the fainter stars) spectra over a limited spectral range, centred on the CaII IR triplet, for stars down to the $17^{\text {th }}$ magnitude.

For the fainter stars there will be no radial velocity information. For all the stars the chemical information will be limited to a few species, and different for different spectral types. For cool stars of types $\mathrm{F}$ to K, these will be limited to $\mathrm{Mg}, \mathrm{Si}, \mathrm{Ca}, \mathrm{Ti}$, and Fe. Such detailed information can be envisaged only for stars brighter than about 12 . For the fainter stars the spectro-photometry will only provide a mean "metallicity". The combination of parallaxes and proper motions will provide transverse velocities with an accuracy better than $1 \mathrm{~km} \mathrm{~s}^{-1}$ for the majority of stars within $1 \mathrm{kpc}$. However, this accuracy will not be matched by the radial velocities provided by the RVS for the stars fainter than about 13. A reliable analysis of the Galactic dynamics requires an accuracy of the order of $1 \mathrm{~km} \mathrm{~s}^{-1}$ for all the components of the velocity.

For all of the above reasons, wide field high resolution spectroscopic surveys from ground based facilities appear as a necessary complement to guarantee the maximum scientific throughput of the Gaia mission. The wide field of view attainable at the prime focus of $4 \mathrm{~m}$ class telescopes makes these an attractive option for placing a multi-object spectrograph. This is in fact the choice already made by the Anglo Australian Telescope, with the HERMES project[1]. The Canada-France-Hawaii Telescope has a long tradition in providing a stable prime focus environment for astronomical instrumentation and it thus appears a natural choice to study the feasibility of multi-object fibre-fed spectrograph for this telescope. The two projects would be strongly complementary since HERMES will have access to the southern hemisphere, while GYES to the northern hemisphere

\section{SCIENCE CASES}

\subsection{Chemical labelling and galactic substructure}

It is now widely recognized that the different populations of Galaxies may present distinct signatures of chemical composition, due to the details of the chemical evolution. The most well known is the distinction in $[\mathrm{Mg} / \mathrm{Fe}]$ between thick and thin disc, first highlighted by Fuhrmann[2]. Vanessa Hill introduced the concept of "chemical 
labelling": when one is able to determine abundance ratios with an error of 0.1 dex for the purpose of putting a chemical "label" on a given stellar population. Even more ambitious is the task of "chemical tagging"[3] which aims at identifying the members of an individual star-forming cloud which would require accuracies of the order of 0.01 dex.

One of the primary aims of GYES is to provide chemical abundances accurate to $\sim 0.1$ dex for the different components of the Galaxy: thin disc, thick disc, bulge, halo. The stellar clusters, both open and globular, are primary targets for GYES. These requirements set immediately the minimal requirements for the resolution of the spectrograph and for the field of view of the instrument.

Experiments with both synthetic spectra and real data convinced us that the spectral resolution should not be less than about 15000 . Multi-element chemical analysis at lower resolution have been conducted[4, 5] these were directed towards metal-poor stars for which line blending is not a major problem. Below the resolution of $\mathrm{R} \sim 15000$ the chemical analysis relies only on strong lines, which are mainly on the flat part of the curve of growth, whichever the $\mathrm{S} / \mathrm{N}$ ratio of the spectra. This implies that their sensitivity to abundance variations is small. To some extent this may be compensated for elements like Fe or Ca, which display a large number of lines, if one may rely on a large wavelength coverage. The uncertainty of the single lines averages out by using many lines; however, for species which rely on few lines this is not possible.

A minimal requirement on the field of view comes from the lower density environment considered: the halo. To derive this requirement we need to have a crude estimate of what can be the performances of a spectrograph at the CFHT. A crude, but robust, estimate can be obtained by scaling the performances of Giraffe at the VLT $[6,7]$. Taking into account the different collecting surfaces of the two telescope one can expect to obtain at CFHT the same performances as Giraffe, for stars which are about 1.8 magnitudes brighter. For Giraffe the exposure time calculator provides a $\mathrm{S} / \mathrm{N}$ ratio of about 30 per pixel at $630 \mathrm{~nm}$ in two hours of exposure for a $\mathrm{K}$ type star of $\mathrm{V}=17.8$. Analysis of Giraffe data of this $\mathrm{S} / \mathrm{N}$ ratio shows that the accuracy in chemical abundances of 0.10 to $0.15 \mathrm{dex}$ is in fact attainable. It is reasonable to expect a similar performance for GYES at the CFHT for a 16th magnitude star. From existing catalogues and models of the Galaxy[8] we can expect to have between 1 and $30 \mathrm{~K}$ giants, depending on Galactic latitude, brighter than $16^{\text {th }}$ magnitude per square degree. Although we will certainly want to be able to observe fainter stars, this figure suggests that we should aim at least at a field of view of one square degree. Clearly this constraint is not tight, a more efficient spectrograph can allow to reach fainter magnitudes at the same $\mathrm{S} / \mathrm{N}$ ratios in the same time.

\section{$2.2 \quad$ Pulsating stars}

In the magnitude range $6<V<20$ Gaia will detect about 190000 pulsating stars showing a Period-Luminosity $(P L)$ relation (35000 RR Lyrae, 5000 Cepheids and $150000 \delta$-Scuti stars[9]).

Once the Gaia parallaxes will be available, these $P L$ relations can be accurately calibrated, including also possible effects of other quantities, such as metallicity. Therefore the goal of GYES is to obtain chemical compositions for these stars. Table 1 gives the corresponding limiting magnitude together with the number of expected pulsating stars observable per square degrees in different directions within the Milky Way.

Table 1: Number of stars per square degrees from the Besançon model[8]. The spectral type, the luminosity and the age used in the simulation are respectively : Cepheids (F2-K2 I-II, 0-0.15 Gyr), $\delta$-Scuti (A2-F2 III-IV, 0.15-1 Gyr) and RR Lyrae (A2-F6 II-III , ages of "intermediate populations, bulge and halo"). The integration time is limited by the short periodicity of RR Lyrae and $\delta$-Scuti stars: it should be lower than a tenth of the period of the star[10].

\begin{tabular}{l|cccc|cccccc}
\hline Stars & Period (d) & $\begin{array}{c}\text { Exposure } \\
\text { Time }\end{array}$ & Magnitude & $\mathrm{S} / \mathrm{N}$ & $\ell=0$ & 90 & 180 & 270 & - & - \\
& & & & $b=0$ & 0 & 0 & 0 & 90 & -90 \\
\hline Cepheids & $2<P<50$ & $t=1 h$ & $6<V<16$ & $>=50$ & 404 & 4 & 1 & 5 & - & - \\
RR Lyrae & $0.2<P<1.2$ & $t=30^{\prime}$ & $6<V<15$ & $>=50$ & - & - & - & - & 0 & 0 \\
$\delta$-Scuti & $0.05<P<0.2$ & $t=15^{\prime}$ & $6<V<14$ & $\geq 50$ & 284 & 161 & 142 & 191 & - & - \\
\hline
\end{tabular}

While it is clear that RR Lyrae are too faint to be an interesting target for GYES, it should be capable to 


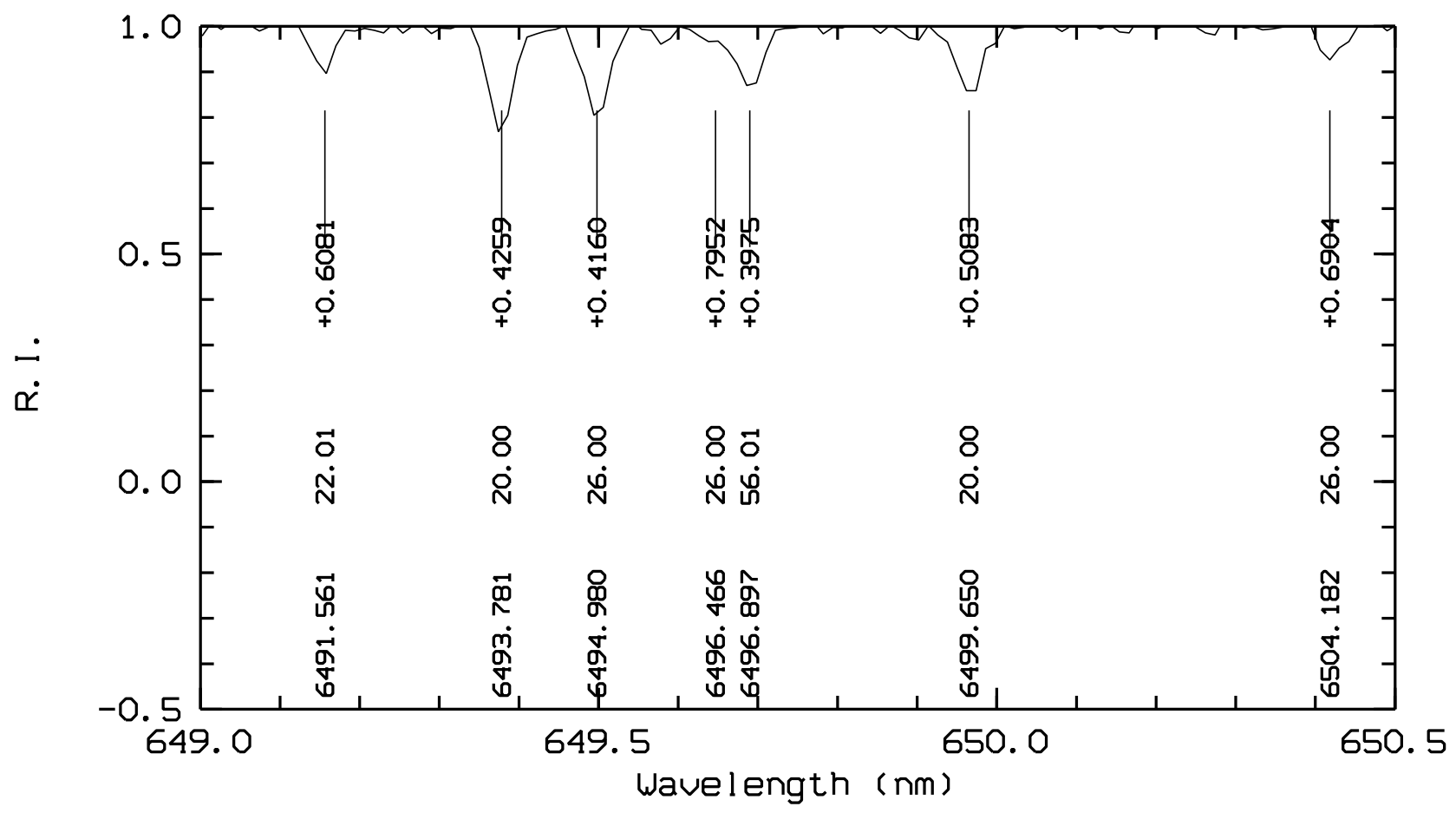

Figure 1: The BaII $649.6 \mathrm{~nm}$ line in a 1D synthetic spectrum of a solar type star, $6000 \mathrm{~K} / 4.0 /-1.0$, convolved with a Gaussian profile of $\mathrm{R}=20000$. Poisson noise has been injected so that $\mathrm{S} / \mathrm{N}=100$.

observe all Cepheids in the northern hemisphere provided by the Gaia catalog (in good conditions: $\mathrm{S} / \mathrm{N}$ better than 50 for a $1 \mathrm{~h}$ integration time), as suggested by the histogram of the apparent brightness of known Galactic Cepheids[11]. It should also be capable to observe $22500 \delta$-Scuti stars in good conditions (S/N better than 50). Observations of these pulsating stars should be scheduled together with the other survey targets.

\section{$2.3 \quad$ Interstellar medium}

In the course of the survey, it will be possible to observe also early type stars (earlier than A) which can be used as background sources to map the Galactic InterStellar Medium (ISM). The most numerous will be hot Horizontal Branch stars.

The warm and cold ISM can be traced by $\mathrm{Ca}$ II $\mathrm{H}$ and $\mathrm{K}$ and $\mathrm{Na}$ I D lines observed in absorption against these background sources. If a spectral resolution as high as 50000 is available, it may be possible to disentangle the absorptions due to different ISM clouds, which differ by only a few $\mathrm{km} \mathrm{s}^{-1}$. At this high resolution it may also be possible to use cooler stars as background sources, provided their radial velocity is high enough to disentangle them from the stellar absorptions. At lower resolutions, like 20000 , it will still be possible to obtain interesting column densities of $\mathrm{Ca}$ II and $\mathrm{Na}$ I which may be used as diagnostics of reddening and detect high velocity clouds.

\subsection{Extragalactic astronomy}

Giraffe at the VLT has demonstrated the flexibility which can be attained by allowing the same spectrograph to be fed by different apertures on the sky, from single-object fibres to integral field units. The latter provide a convenient way of doing 3D spectroscopy. In order to do interesting science in the field of extragalactic astronomy we require that GYES has available a lower resolution mode (R 1000/3000) and a large integral field unit. 
Table 2: Spectral content of the proposed wavelength ranges. The list is not exhaustive, but lists some of the main spectral features that can be found in the spectra of stars of spectral type $\mathrm{K}$ to $\mathrm{B}$.

$[380 ; 450] \mathrm{nm}$ domain

$[580 ; 680] \mathrm{nm}$ domain

\begin{tabular}{|c|c|c|c|c|c|c|c|}
\hline Parameter & Element & \# lines & $\begin{array}{l}\text { Wavelength } \\
{[\mathrm{nm}]}\end{array}$ & Element & \# lines & $\begin{array}{c}\text { Wavelength } \\
{[\mathrm{nm}]}\end{array}$ & Comment \\
\hline \multirow{3}{*}{$T_{\text {eff }}$} & FeI & Many & & FeI & 26 & & \\
\hline & $\mathrm{H} \delta$ & 1 & 410.1 & $\mathrm{H} \alpha$ & 1 & 656.5 & \\
\hline & $\mathrm{H} \gamma$ & 1 & 434.1 & & & & \\
\hline \multirow[t]{3}{*}{$\log g$} & \multirow[t]{3}{*}{ FeII } & \multirow[t]{3}{*}{8} & \multirow{3}{*}{$\begin{array}{c}382.4,407.5,417.8 \\
423.3,429.6,430.3 \\
441.6,449.1\end{array}$} & FeII & 2 & $645.6,667.7$ & \\
\hline & & & & FeII & 5 & & weak lines \\
\hline & & & & FeI & 26 & & \\
\hline \multirow[t]{6}{*}{ Helium } & HeI & 1 & 400.9 & \multirow[t]{6}{*}{ HeI } & \multirow[t]{6}{*}{1} & \multirow[t]{6}{*}{667.8} & \\
\hline & HeI & 1 & 402.6 & & & & \\
\hline & HeI & 1 & 414.4 & & & & \\
\hline & HeI & 1 & 438.7 & & & & \\
\hline & He I & 1 & 447.1 & & & & \\
\hline & HeII & 1 & 420.0 & & & & \\
\hline$v \sin i$ & MgII & 2 & 448.1 & & & & \\
\hline$[\mathrm{Fe} / \mathrm{H}]$ & FeI & Many & & FeI & 26 & & \\
\hline \multirow[t]{5}{*}[\alpha/\mathrm{Fe}]{} & CaI & 12 & \multirow{5}{*}{$\begin{array}{c}382.9,416.7 \\
390.5\end{array}$} & $\mathrm{CaI}$ & 12 & & \\
\hline & MgI & 2 & & MgI & 2 & & weak features \\
\hline & SiI & 1 & & SiI & 8 & & \\
\hline & TiI & 5 & & SiI & 7 & & weak feature \\
\hline & TiII & 10 & & TiI & 5 & & weak lines \\
\hline \multirow[t]{11}{*}[\mathrm{X}/\mathrm{Fe}]{} & VI & 2 & \multirow{11}{*}{407.8} & VI & 1 & 609.0 & weak line \\
\hline & VII & 1 & & MnI & 1 & 601.3 & weak line \\
\hline & MnI & 8 & & $\mathrm{NaI}$ & 1 & 615.4 & weak line \\
\hline & ScI & 1 & & BaII & 1 & 649.6 & strong line \\
\hline & ScII & 3 & & ScII & 1 & 660.4 & weak line \\
\hline & AlI & 1 & & AlI & 1 & 669.6 & weak line \\
\hline & CrI & 5 & & LiI & 1 & 670.8 & \\
\hline & CoI & 3 & & & & & \\
\hline & YII & 2 & & & & & \\
\hline & SrII & 1 & & & & & \\
\hline & $\mathrm{CH}$ & 1 & & & & & \\
\hline
\end{tabular}




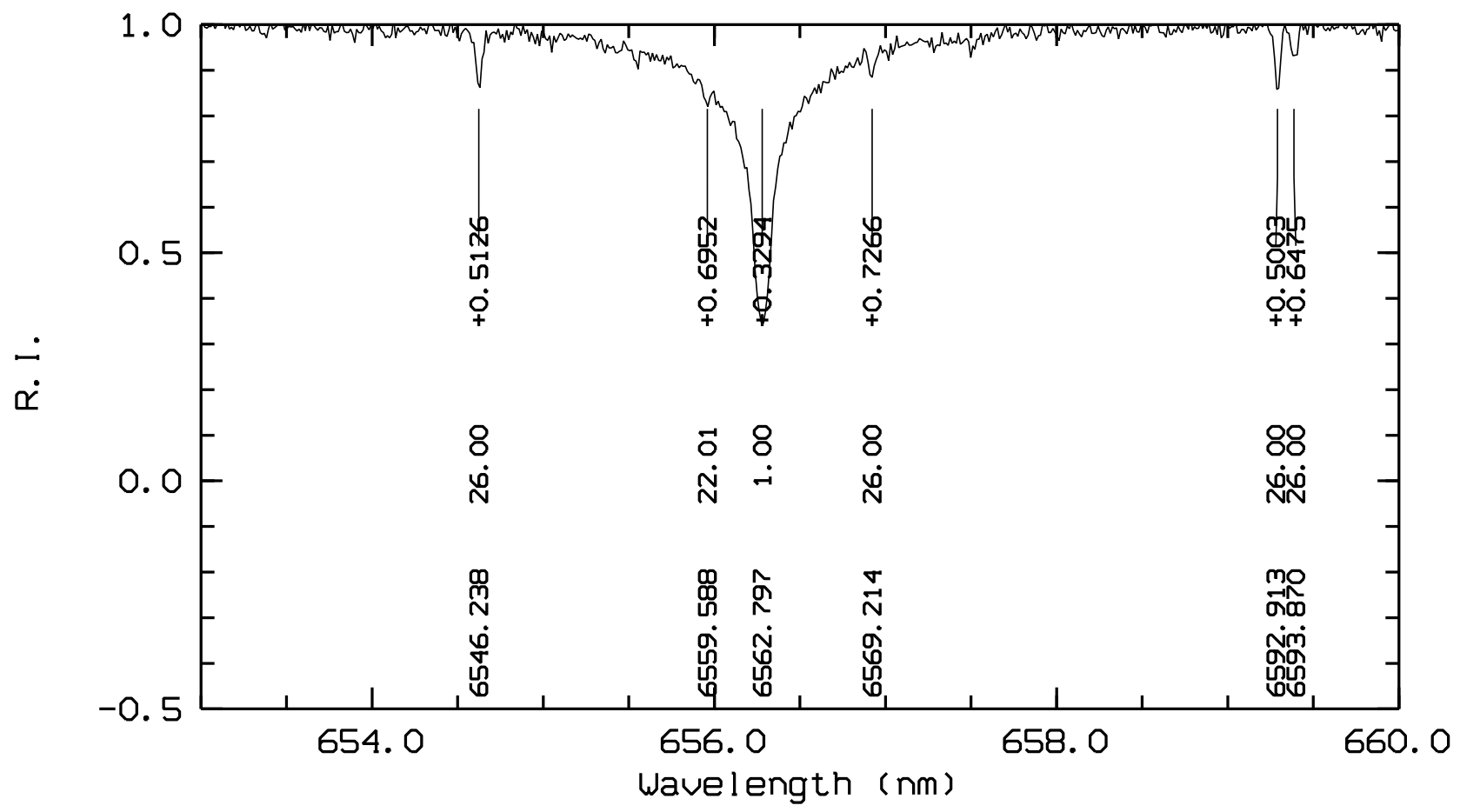

Figure 2: $\mathrm{H} \alpha$ line profile in a $1 \mathrm{D}$ synthetic spectrum of a solar type star, $6000 \mathrm{~K} / 4.0 /-1.0$, convolved with a Gaussian profile of $\mathrm{R}=20000$. Poisson noise has been injected so that $\mathrm{S} / \mathrm{N}=100$.

In spite of the large advances in available data for imaging of local galaxies (for example SDSS[12], 2MASS[13], GALEX[14]) the number of surveys with spatially resolved spectroscopy is still small. A considerable wealth of information on the local Universe $(z<0.05)$ can be derived from space-resolved spectroscopy. In particular it is possible to map across a galaxy: $i$ ) the properties of its ISM (metallicity, electronic temperature, density, extinction); ii) kinematics of the ionised gas and of the stars. The IFU can allow to map the behaviour of emission and absorption lines in the galactic spectra. The sizes of local galaxies, and their extended gaseous halos prompt for an IFU size of $1 \operatorname{arcmin}^{2}$, or larger. On the other hand the spatial sampling can be of the order of $2^{\prime \prime} .5$, leading to an array of the order of 600 spaxels.

The spectral features of galaxy spectra to be studied are the Balmer break, the G band, the Mg I b triplet, in absorption, the Balmer lines, in emission or absorption, depending on the galaxy type, [OII], [OIII], [NII], and [SII] lines, in emission. These tracers suggest two useful spectral ranges, one in the blue between 370 and $530 \mathrm{~nm}$ and another in the red between 650 and $720 \mathrm{~nm}$.

\section{STELLAR CHEMICAL ANALYSIS}

With an average exposure time of $2 \mathrm{~h}$, and a spectrograph of 500 fibers, 400 of which pointed on a star, and the rest on the sky, we expect to observe about 1500 targets in one night. This is far too much for a traditional analysis. We plan to analyse automatically the stellar spectra. Automatic codes for abundance determination based on $\chi^{2}$ fitting [15] or sophisticated algorithms such as MATISSE[16] are already available and can be used for the purpose.

The analysis may be based on 1D, plane parallel, LTE model atmosphere, but for some elements, and particularly in metal-poor stars, we should not forget the granulation effects on the abundance determination. The average temperature structure of a 3D metal-poor model is cooler in the external layers with respect to a traditional 1D model atmosphere. The spectral lines that are formed in the external photosphere $(\log \tau \lesssim-2)$ are much affected by this cooling. The hydrodynamical models follow the gas that flow in the box located 


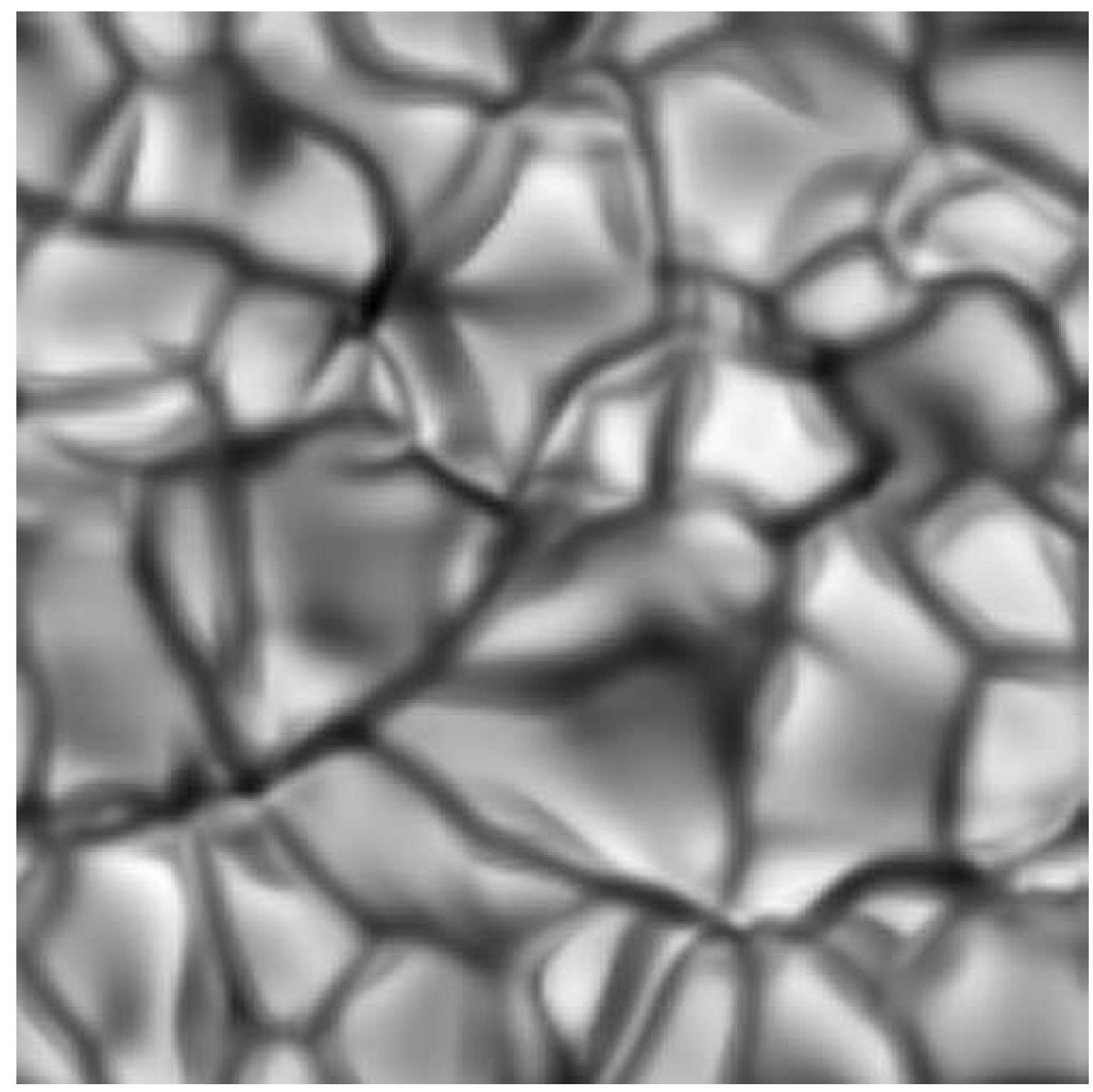

Figure 3: Emergent continuum intensity for the 3D model $4500 \mathrm{~K} / 2.5 / 0.0$ 
in the stellar photosphere, are able to reproduce the stellar granulation so typical in the solar images. In Fig. 3 the emergent continuum intensity, for a $3 \mathrm{D}$ model of parameters $4500 \mathrm{~K} / 2.5 / 0.0$, is shown, to point out the granulation pattern in a cool star. In fact a fairly complete grid of hydrodynamical models already exists[17] and by the time GYES will be on the sky one can expect to be able to perform the full analysis using hydrodynamical models.

\section{SCIENCE REQUIREMENTS}

\begin{tabular}{llrrrr}
\hline \hline \multicolumn{2}{l}{ Science case } & \multicolumn{1}{c}{$R$} & \# fibres & $V$ range & RV accuracy \\
\hline Sect. 2.1 & Thin and thick discs & 20000 & $200-500$ & $10-16$ & $1-5$ \\
& Bulge & 20000 & 500 & $15-17.5$ & 1 \\
& Halo & 20000 & 300 & $18-20$ & $1-10$ \\
& Open clusters & $20000-40000$ & $20-30$ & $10-16$ & 0.1 \\
& Globular clusters & $10000-40000$ & 200 & $16-20$ & $1-5$ \\
\hline Sect. 2.2 & $20000-30000$ & 12 & $6-16$ & 1 \\
\hline Sect. 2.3 & 50000 & $>100$ & - & - \\
\hline Sect. 2.4 & $1000-3000$ & 600 & - & - \\
\hline
\end{tabular}

Table 3: Summary of the requirements for the different science cases. The science cacses are identified by their corresponding section number in the paper: (2.1) Chemical labelling and galactic substructures; (2.2) Pulsating stars; (2.3) Interstellar medium (2.4) Extragalactic astronomy. $V$ range is the expected magnitudes of the targets and RV accuracy is the needed accuracy of the radial velocities expressed in $\mathrm{km} \mathrm{s}^{-1}$.

The science requirements follow from the aforementioned science cases. The requirements from individual science cases are listed in Table 3. The primary scientific objective of GYES is to high resolution spectra for a very large number of Gaia targeted stars in order to assess their precise radial velocities and chemical abundances.

\subsection{Spectral ranges}

The choice of the spectral range is driven by scientific expectations of GYES. We want to extract sufficient informations from the spectra. For the measurement of radial velocity any spectral range with a large enough number of lines can be satisfactory.

For the chemical labelling we need to derive, at least, temperature, gravity, $[\mathrm{Fe} / \mathrm{H}]$, and $[\alpha / \mathrm{Fe}]$, so that the spectral range should contain the relevant lines; in addition we would like to determine chemical abundances for as many elements as possible. In the following we shall focus on the analysis of cool stars which constitute the majority of the surveyed stars. For temperature, gravity, and metallicity determination Fe I lines are necessary. We need FeII lines for the gravity determination, and lines of an $\alpha$ element (e.g. $\mathrm{Ca}, \mathrm{Mg}$ ) for the $\alpha$ enhancement determination. Any other element present in the range permits to add chemical informations on the targets. We selected two ranges, at $[380-450] \mathrm{nm}$ and $[580-680] \mathrm{nm}$, to fulfill all our request. On top of that some interesting elements have at least one line in the ranges, such as $\mathrm{Li}, \mathrm{Ba}$, and Al. A schematic summary is shown in Table 2. It is interesting to note that all the main nucleosynthetic channels are probed by the proposed wavelength ranges: i) nuclear statistical equilibrium (NSE), iron peak elements; ii) $\alpha$-chain (Mg, Si, Ca, Ti); iii) neutron capture $(\mathrm{Sr}, \mathrm{Y}, \mathrm{Ba})$; iv) proton capture $(\mathrm{Al}, \mathrm{Na})$.

In the red range $\mathrm{H} \alpha$ line is present. The wings of this strong hydrogen line (see Fig. 2) are sensitive to the temperature $[18,19,20]$. We can fit $\mathrm{H} \alpha$ wings to derive the stellar effective temperature, the higher members of the series $\mathrm{H} \gamma, \mathrm{H} \delta$ can also be used for this purpose. Their use is somewhat more difficult, because of blending of metallic lines but they can be used to support the $\mathrm{H} \alpha$ analysis. The sensitivity of the higher members of the Balmer series to the treatment of convection[18] is not an issue if one makes use of three dimensional hydrodynamical simulations[20]. 
For hot stars of type B the proposed ranges allow access to several Balmer lines, and for this spectral type the wings are sensitive to surface gravity, and are also important diagnostic of emission-line stars and for their circumstellar disc modelling. The helium lines can be used to constrain the effective temperatures. The MgII $448.1 \mathrm{~nm}$ doublet is often useful to determine the projected rotational velocities.

\subsection{Spectral resolution}

A mean resolution of 20000 fulfills most of the science cases previously mentioned. As far as the interstellar medium is concerned (2.3), such a resolution does not prevent the feasibility of the science case.

The resolution should not get below 15000 anywhere over the spectral ranges, in nominal conditions.

\subsection{Efficiency}

The main aim is to provide an instrument capable of performing a spectroscopic survey complementary to Gaia. We envisage a survey which may obtain spectra for about one million stars, down to 18th magnitude. We target an efficiency such that a signal-to-noise ratio $\mathrm{S} / \mathrm{N}=30$ is obtained in a 2 hour integration for a 16th magnitude star.

\subsection{Multiplex}

The multiplex should be of the order of 500 fibres. It would ensure to cover the high stellar density regions in the Galactic disk and bulge and to dedicate a sufficient number of fibres to blank sky positions for sky subtraction purposes.

\subsection{IFU}

In addition to the regular fibre system deployable on individual targets, one could add also an IFU and a low resolution mode $(R=1000-3000)$ that can be used for area spectroscopy of extended objects $\left(\sim 1^{\prime}\right)$. This is considered as a possible option.

\subsection{Calibration system}

GYES shall be equipped with its own calibration system, to perform scientific and technical calibrations in order to remove the instrument signature and monitor the instrument.

\section{ACKNOWLEDGMENTS}

The GYES project is funded by CNRS, Institut National Science de l'Univers and by Observatoire de Paris.

\section{References}

[1] Wylie-de Boer, E. and Freeman, K., "HERMES - An instrument of the future," in [Stellar Populations Planning for the Next Decade], G. Bruzual \& S. Charlot, ed., IAU Symposium 262, 448-449 (Apr. 2010).

[2] Fuhrmann, K., "Nearby stars of the Galactic disk and halo," A\&A 338, 161-183 (Oct. 1998).

[3] Freeman, K. and Bland-Hawthorn, J., "The New Galaxy: Signatures of Its Formation," ARAEA 40, $487-537$ (2002).

[4] Lai, D. K., Bolte, M., Johnson, J. A., and Lucatello, S., "Abundances of Extremely Metal-poor Star Candidates," $A J$ 128, 2402-2419 (Nov. 2004).

[5] Kirby, E. N., Guhathakurta, P., Bolte, M., Sneden, C., and Geha, M. C., "Multi-element Abundance Measurements from Medium-resolution Spectra. I. The Sculptor Dwarf Spheroidal Galaxy," ApJ 705, 328-346 (Nov. 2009). 
[6] Hammer, F., Hill, V., and Cayatte, V., "GIRAFFE sur le VLT: un instrument dédié à la physique stellaire et extragalactique.," Journal des Astronomes Francais 60, 19-25 (Dec. 1999).

[7] Pasquini, L., Avila, G., Blecha, A., Cacciari, C., Cayatte, V., Colless, M., Damiani, F., de Propris, R., Dekker, H., di Marcantonio, P., Farrell, T., Gillingham, P., Guinouard, I., Hammer, F., Kaufer, A., Hill, V., Marteaud, M., Modigliani, A., Mulas, G., North, P., Popovic, D., Rossetti, E., Royer, F., Santin, P., Schmutzer, R., Simond, G., Vola, P., Waller, L., and Zoccali, M., "Installation and commissioning of FLAMES, the VLT Multifibre Facility," The Messenger 110, 1-9 (Dec. 2002).

[8] Robin, A. C., Reylé, C., Derrière, S., and Picaud, S., "A synthetic view on structure and evolution of the Milky Way," AEA 409, 523-540 (Oct. 2003).

[9] Eyer, L. and Cuypers, J., "Predictions on the Number of Variable Stars for the GAIA Space Mission and for Surveys such as the Ground-Based International Liquid Mirror Telescope," in [IAU Colloq. 176: The Impact of Large-Scale Surveys on Pulsating Star Research], L. Szabados \& D. Kurtz, ed., Astronomical Society of the Pacific Conference Series 203, 71-72 (2000).

[10] Takeda, Y., Honda, S., Aoki, W., Takada-Hidai, M., Zhao, G., Chen, Y., and Shi, J., "On the Spectroscopic Determination of Atmospheric Parameters and O/Fe Abundances of RR Lyrae Stars," PASJ 58, 389-406 (Apr. 2006).

[11] Szabados, L., "Database on Binaries among Galactic Classical Cepheids," Information Bulletin on Variable Stars 5394, 1-+ (Mar. 2003).

[12] Abazajian, K. N. et al., "The Seventh Data Release of the Sloan Digital Sky Survey," ApJS 182, 543-558 (June 2009).

[13] Skrutskie, M. F. et al., "The Two Micron All Sky Survey (2MASS)," AJ 131, 1163-1183 (Feb. 2006).

[14] Martin, D. C. et al., "The Galaxy Evolution Explorer: A Space Ultraviolet Survey Mission," ApJ 619, L1-L6 (Jan. 2005).

[15] Bonifacio, P. and Caffau, E., "Automatic abundance analysis of high resolution spectra," $A \& A$ A99, 11831192 (Mar. 2003).

[16] Recio-Blanco, A., Bijaoui, A., and de Laverny, P., "Automated derivation of stellar atmospheric parameters and chemical abundances: the MATISSE algorithm," MNRAS 370, 141-150 (July 2006).

[17] Ludwig, H., Caffau, E., Steffen, M., Freytag, B., Bonifacio, P., and Kučinskas, A., "The CIFIST 3D model atmosphere grid.," Memorie della Società Astronomica Italiana 80, 711-+ (2009).

[18] Fuhrmann, K., Axer, M., and Gehren, T., "Balmer lines in cool dwarf stars. 1. Basic influence of atmospheric models," A\&A 271, 451-+ (Apr. 1993).

[19] Barklem, P. S., Stempels, H. C., Allende Prieto, C., Kochukhov, O. P., Piskunov, N., and O’Mara, B. J., "Detailed analysis of Balmer lines in cool dwarf stars," A $E A$ 385, 951-967 (Apr. 2002).

[20] Ludwig, H., Behara, N. T., Steffen, M., and Bonifacio, P., "Impact of granulation effects on the use of Balmer lines as temperature indicators," A\&A 502, L1-L4 (Aug. 2009). 